\title{
A Framework for Intelligent Inventory Prediction in Small and Medium- Scale Enterprise
}

\author{
Okure Obot $^{1 *} \quad$ Uduak George $^{1} \quad$ Imaobong James ${ }^{2}$ \\ 1. Department of Computer Science, University of Uyo, Uyo. Nigeria \\ 2.Aberdeen Business School, Robert Gordon University, Aberdeen, United Kingdom
}

\begin{abstract}
The aim of this research is to apply an intelligent technique to predict optimal inventory quantity in small and medium-scale enterprise. This is in view of the fact that the conventional models such as the EOQ model use only deterministic while some decision variables are non- deterministic in nature. Forecasted average demand of items for ten months in a small-scale retail outlet was collected and trained using an Artificial Neural Networks (ANN) of 5 neurons in the input layer with eight neurons in the first hidden layer and four neurons in the second hidden layer. Two feed-forward training algorithms of quasi-newton and quick propagation were employed in the training with the results of fuzzy logic technology found in the literature as the target output. Results obtained show that the quasi-newton algorithm covaries stronger with the fuzzy logic results than the quick propagation results. The objective and subjective feelings of the inventory manager were also captured to optimise the results of the training. The study is at a framework stage and will proceed to implementation level when more datasets are collected. Data collection in a small-scale outlet is a daunting task as record keeping is hardly done. The inclusion of nondeterministic circumstances such as emotional and objective feelings of the inventory manager to predict inventory is novel considering the fact that studies in the available intelligent inventory prediction have not employed such variables in their predictions.
\end{abstract}

Keywords: Artificial Neural networks, Fuzzy logic, Quasi newton, Quick propagation, EOQ, Inventory, Forecast. DOI: $10.7176 / \mathrm{EJBM} / 13-2-03$

Publication date: January $31^{\text {st }} 2021$

\subsection{Introduction}

Small and medium scale enterprises are wont of carrying a large amount of inventory, mainly if stocks are produced from a long-distance industry. This, they do to avoid harming customers' goodwill by ensuring a constant supply of goods and services. In doing so, the cost of storage and the risk of obsolescence and deterioration are incurred. If what is carried at a time is not enough relative to demand, the risk of losing customers' goodwill could lead to substantial loss. According to Pan (2016), predicting inventory can improve the ability of enterprises to prevent risk, increase profits and reduce losses. The trade-off between incurring cost due to large quantity of inventory and losing customers' goodwill due to lack of an item on demand has led to developing optimisation tools to predict optimal quantity to carry and keep at a time. One of such tools is the Economic Order Quantity (EOQ) model. Attempts to modify the EOQ model are made in various studies including Obot et al. (2019), where a decision support tool that merges fuzzy logic technology with the EOQ model is developed.

Effective inventory management is an essential part of every business to avoid the cost associated with backorder penalties during different demand frequencies and storage time which often leads to business failures. Intelligent decision support system is capable of processing vague and uncertain data that affect carrying and holding cost of inventory. Small and medium scale businesses in Nigeria are mostly privately-owned enterprises that foster the acceleration of economic development and technological progress of the nation. In Nigeria, small and medium scale businesses constitute over 90 per cent of all the companies and contribute immensely to the generation of employment, provision of goods and services and adds to the gross domestic products (GDPs) (Kehinde et al. 2016; Motilewa, 2015; Ogundele et al. 2013; SMEDAN 2011).

Although small and medium scale businesses contribute mainly to the nation's economy, they are faced with the different challenges which include limited impact in the market place, limited resources such as finances, expertise and limited demands for their goods and services (Basil 2005; Damirchi and Rahimi 2011). Other factors that impacts on Nigerian small and medium scale businesses include unfavourable government and economic policies due to political instabilities, poor access to funds which can lead to limited business growth and performance (Esther et al. 2018; Agwu and Emeti 2014; Taiwo et al. 2012; Oluboba 2011).

Research has shown that small and medium scale businesses go out of business within their first five years and only about five to ten per cent of small and medium scale businesses in Nigeria survive to maturity (Eze and Okpala 2015; Ihua 2009). This study suggests that small and medium scale businesses can overcome the challenges associated with inventory management cost. The inventory prediction system will enable small and medium scale businesses, especially those operating as retail outlets to reduce operational cost, increase sales thereby decreasing their risk of failure and increasing their chances of survival and contributions to the nation.

The current method where small and medium scale businesses in the retail outlets are using to process stock 
based on previous sales data and reorder point comes with a high cost of carrying and holding inventory. This study is based on the data obtained from a supermarket in Ikot Ekpene in Nigeria on fifteen items consisting of 5 seasonal items and ten non-seasonal items. Information from the data includes quantity of daily sales, the unit price, the lead time and the frequency of the order of each item in a month. These data enabled the development of the proposed Economic Order Quantity (EOQ) method, which will enable retail outlets to overcome these inadequacies and optimise resources while minimising inventory cost based on daily predictions. EOQ model has been known to deal on only deterministic data. Obot et al. (2019) show that certain variables that affect holding and carrying cost are fuzzy. Fuzzy logic and neural network technology have been known to handle data with both a linear and non-linear relationship. This study attempts to develop a reliable, intelligent decision-support system which is capable of minimising cost and maximising inventory turn-over ratios while ensuring customers' needs are satisfied, and the organisation is making a profit.

\subsection{Literature Review}

Every organisation requires inventory which includes the raw materials, work-in-process, supplies used in operations and finished products in retail outlets/distribution sectors. Inventory management involves all activities in developing and managing the inventory level of raw material, semi-finished and finished goods such that adequate supplies are available at a reduced cost (Kotler, 2000). It involves the coordination of materials and products accessibility, controlling, utilisation and procurement of both physical items and intangible goods such as record counts and monetary worth (Muller, 2019). Many organisations depend mainly on the inventory control system for their profitability. Inventory consists of the following number of costs: money, space, labour to receive, check the quality and quantity, put away deterioration, damage, obsolescence and theft. Generally, inventory cost usually falls under ordering costs and holding costs. There are various reasons some organisations, especially small and medium scale businesses, engage in obtaining and holding inventory which includes: predictability, fluctuation in demand, unreliability in supply, price protection and quantity discounts.

There are different types of inventory. This study focuses on the inventory of finished goods ready for customer sales. Effective inventory management system enables the understanding of the modern practice that discourages the holding of the excess quantity of goods and encourages the required amount in stock for the current needs of the customers (Muller, 2019). The inventory control system also applies formulae for calculating inventory quantities and select counting inventory method that is right for the organisation. The development of laser technology has enabled faster and cheaper reader or scanners with the use of Universal Product Code to help track and manage inventory in the developed economy. Product information such as type, serial numbers are transmitted by Radio Frequency Identification (RFID) with the use of microchips to a scanner and other data collection devices (Agha, 2010). In Nigeria, especially in small and medium scale businesses with limited staff strength and involvement with simple inventory management system due to factors such as lack of infrastructures, economy instabilities, inadequate network systems.

An effective inventory system is made of many features such as simplified implementation, service quality and cost-effectiveness (Kumur and Kumar, 2017). Sekar and Uthayakumar (2018) developed a production inventory model for a single vendor and single buyer integrated demand with multiple production setups and rework. Anwana and Nyah (2017), Gonzalez and Gonzalez (2017) and Agrawal (2014) used the EOQ model to compute inventory predictions. Fuzzy inventory system is developed in Kosinski et al. (2013), where some level of intelligence is built into their systems.

One characteristic of intelligence is the ability to learn new concepts and tasks. This is exhibited through the biological nervous system whose centre is in the brain. The central nervous system is replicated artificially in the computer system through Artificial Neural Networks (ANN). ANN simulates the behaviour of the human neurons to process information and has found application in almost every facet of life, including prediction. A brief form of ANN-based forecasting for inventory control was undertaken by Bansal et al. (1997). Farhat and Owayjan (2017) developed ERP neural networks inventory control where a prediction of goods needed for an enterprise inventory depends on past sales and some time series events were undertaken. Akay et al (2011) developed an ANN based model of predicting $\mathrm{VO}_{2}$ max from a submaximal exercise test. Paul and Azeem (2011) developed an ANN model for optimisation of finished goods inventory where four inputs (product demand, setup, holding and material costs) were fed into a feed-forward network of 10 hidden layers nodes and one output. Results obtained after 56 epochs show a mean square error of 0.0001 and a correlation of 0.9487 . Dong and Wen (2016) reported improved neural networks prediction model and its application in a supply chain. A comparison of the results of a feed-forward multilayer perceptron (MLP) neural networks with that of recurrent neural networks was undertaken using simulated time-series data and practical sales data. There is an improved prediction accuracy with the recurrent networks over the MLP. An inventory prediction research based on the enhanced Back Propagation (BP) neural networks algorithm was developed by Pan (2016). An improved Gravitational Search Algorithm (GSA) was employed to optimise the parameters of Back Propagation (BP) neural networks algorithm resulting in an improvement of $5.4 \%$ with the BP having an error of $7.11 \%$ over $1.31 \%$ of GSA. 
In Boru et al. (2019) a novel hybrid methodology based on artificial intelligence was proposed for inventory routing problem. Motivation for the research arose from the fact that with expanding customer expectations and growing markets in the competitive business world, some businesses are bound to collapse. The research aimed at obtaining a better and new approach for solving inventory routing problems. The methodology involved a twophased approach where the first approach employed a non-dominated sorting genetic algorithm II simulation while the second approach used neural network to adjust the re-order point and forecasting of the demand at each replenishment time. The research offer opportunity to improve the average service level under uncertain conditions. In the future, other artificial intelligence methods such as particle swarm optimization can be used to set parameters of the neural network.

Llave (2017) presented a systematic literature review on Business Intelligence and Analytics (BI\&A) in small and medium-sized enterprises. It was observed from literature that BI\&A seldom applies to Small and Mediumsized Enterprises (SMEs) which contributes to about $90 \%$ of businesses and more than $50 \%$ of employments in the society, rather it has been generally implemented in large enterprises. In order to make informed business decisions using real-time data, business intelligence (which is a set of methods, processes, architectures and technologies that transform raw data to useful information) should be adopted to improve the performance of SMEs. The research addressed BI\&A components, solutions, applications, adoption, implementation as well as mobile and cloud BI\&A.

Jablonska and Polkowski (2017) presented an artificial intelligence-based processes in Small and Mediumscale Enterprises (SMEs). Huge data generated by SMEs in recent time calls for ways of handling it effectively to achieve effectiveness in the competitive world. The research explored possibilities of Artificial Intelligence (AI) integration into SMEs. AI-based process implementation assists in improving sales, problem solving capacity, organizational performance, lower costs, human workload reduction and cheaper labor. Several implementations of intelligent systems were highlighted though they were all applied in big businesses. The paper however suggested possible intelligent system solutions that could be used in SMEs to include deep learning, speech and language recognition, customer targeting, chatbots and dynamic pricing. The identified challenge of using intelligent systems in SMEs is adequate data, since such systems rely on data for effective performance.

Schuller and Doubravsky (2019) presented fuzzy similarity used in micro-enterprises in marketing communication for sustainable development. Sustainability of a small business is a big challenge in businesses observed by the researchers. Fuzzy reasoning was proposed as a formal tool used to determine the differences in perception of individual communication channels by customers. Fuzzy logic tool was used to represent the linguistic variables based on the assessment of eight marketing communication (MC) channels by five groups of people based on age. The MC were e-mail, social networks, web pages, text message, newspapers, phone calls, posters and radio while the age groups were $<18,18-28,29-39,40-50$ and $>50$. Pairwise fuzzy similarity graphs assisted in determining the relationship between the assessments of a pair of MC by a certain age group of respondents. Subsequent researches could focus on how to use the fuzzy logic tool to determine the sustainable marketing communication channel most relevant to the customers.

Zigiene et al. (2019) presented artificial intelligence based risk management framework for SMEs for risk prevention in SMEs as large scale external and internal data are generated. The research strongly considers the integration of artificial intelligence and machine learning to process large scale unstructured data with its attendant incompleteness and uncertainty in order to improve risk management.

\subsection{Design}

The architecture of the design is depicted in Figure 1 and consists of the inventory datasets as obtained from a small and medium scale business retail outlet which comprises the quantity of items ordered, the average demand of the items for ten months, the carrying cost and the holding cost. The knowledge base consists of the database and the ANN model. The filter engine filters objective and subjective feelings of the inventory manager to complement the results obtained from processing the knowledge base. 


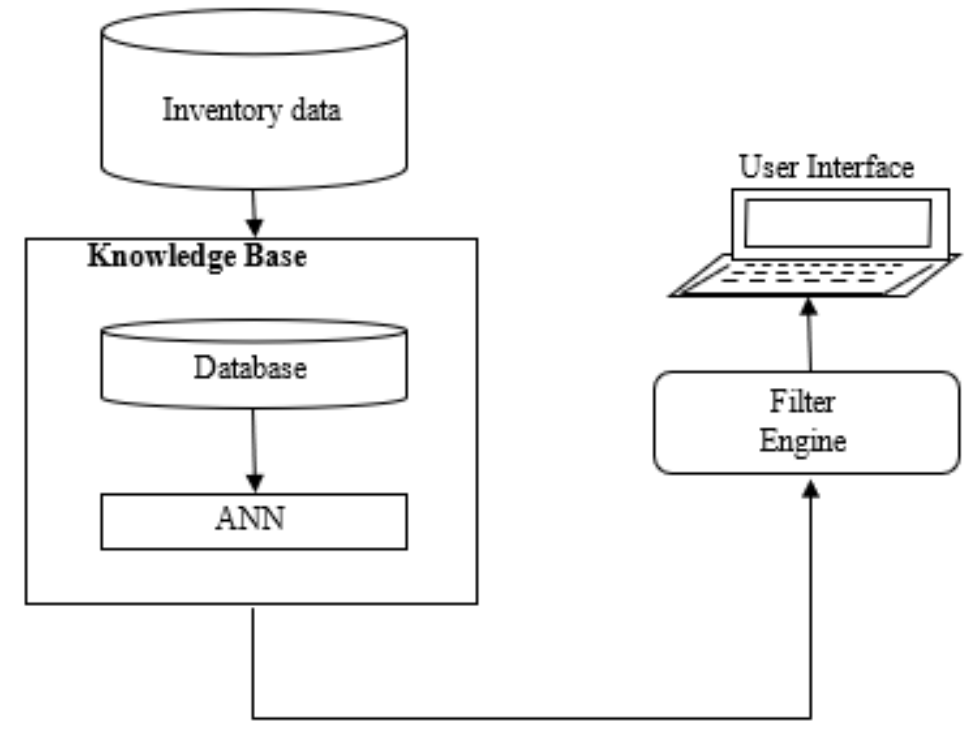

Figure 1: The Design Architecture

\subsection{The Database}

The database is conceptualized as related objects with the attributes of each object depicted as follows Obot et al. (2019):

Cost (Item_Id, Item_Name, Order_Cost, Holding_Cost, Rate_Of_Demand)

ROP (Item_Id, Item_Name, Quantity_Ordered, Quantity_Left, Date_Of_Order, Current_Date, Lead time)

\subsection{The Artificial Neural Network}

A feed forward ANN is trained using two algorithms namely, the Quasi-newton and quick propagation algorithms. Quasi-Newton is a network training algorithm that is based on Newton's method which computes an approximate Hessian matrix at each iteration of the algorithm. Neuro-Intelligence uses the Broyden-Fletcher-Goldfarb-Shanno (BFGS) method for the algorithm (Ramli et al. 2017). Quick propagation algorithm is a modification of the back propagation algorithm. The algorithm considers the weights as quasi-independent and uses a simple quadratic model to approximate the error surface. The structure of the ANN is presented in Figure 2.

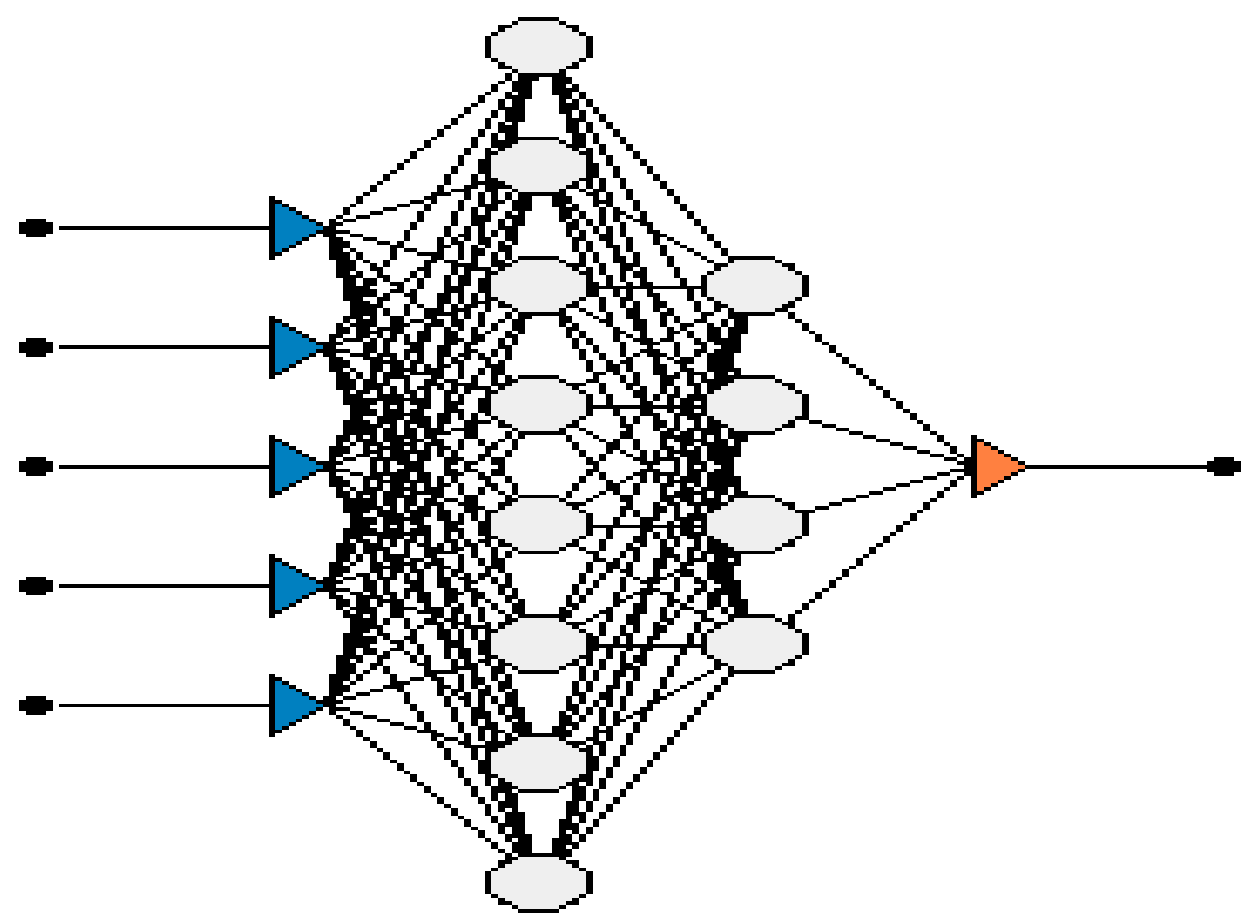

Figure 2: The Artificial Neural Network Architecture for Inventory Prediction 


\subsection{The Filter Engine}

The filter engine comprises the cognitive and emotional filters. The cognitive filter specifies the objective feelings of the inventory manager while the emotional filter specifies the subjective feelings of circumstances.

In the context of this study, the cognitive filter takes into consideration the following scenario:

- The decision to classify an item for inventory taking as very risky, moderately risky and low risky in terms of their deteriorating or obsolete values.

- Lead time, that is a time between when an item is ordered, and when it is delivered could be altered due to urgent needs or scarcity of the item.

- Scarcity of an item due to an unforeseen circumstance such an increase in demand, drop in the supply of raw material used in production, workers being out of service.

The emotional filters include the following:

- Sudden closure of transportation access causing a diversion to a long-distance route with an increasing cost.

- Festivities in the community that can cause a drop or an increase in demand of a product.

- Sudden government policy that could reduce or increase demand of an item.

- Any feelings of expectation that is likely to affect product demand negatively or positively.

\subsection{The Experiment}

A supervised Artificial Neural Network (ANN) learning was carried out using Alyuda Neuro-intelligence software. The best architecture consisting of one input layer, two hidden layers and one output layer was used with five input nodes, 8-first hidden layer nodes, 4-second hidden layer nodes and one node at the output layer. Several network training algorithms were tested, and their results observed. Five attributes were used as inputs namely; Forecast Demand, Unit Cost, Holding Cost, Ordering Cost and Lead Time (days) while Fuzzy EOQ results as found in (Obot et al. 2019) was the target output.

\subsection{Results and Analysis of Findings}

Two training algorithm results were obtained, and observations show that Quasi-Newton provided more satisfactory results. Table 1 shows the results for the Quasi-Newton algorithm compared to its closest rival, Quick Propagation.

Table 1: Training Results

\begin{tabular}{|l|l|}
\hline \multicolumn{2}{|l|}{ Training Algorithm Error Values } \\
\hline $\begin{array}{l}\text { Quasi- } \\
\text { Newton }\end{array}$ & $\begin{array}{l}\text { Quick } \\
\text { Propagation }\end{array}$ \\
\hline 0.057269 & 0.26925 \\
0.006221 & 0.28224 \\
0.089545 & 0.254142 \\
0.040266 & 0.012776 \\
0.063327 & 0.036776 \\
0.04641 & 0.165127 \\
0.031215 & 0.008442 \\
0.200511 & 0.022767 \\
0.247961 & 0.448236 \\
0.015631 & 0.258633 \\
0.04558 & 0.019947 \\
0.081042 & 0.05356 \\
0.025209 & 0.000863 \\
0.002927 & 0.029899 \\
\hline
\end{tabular}




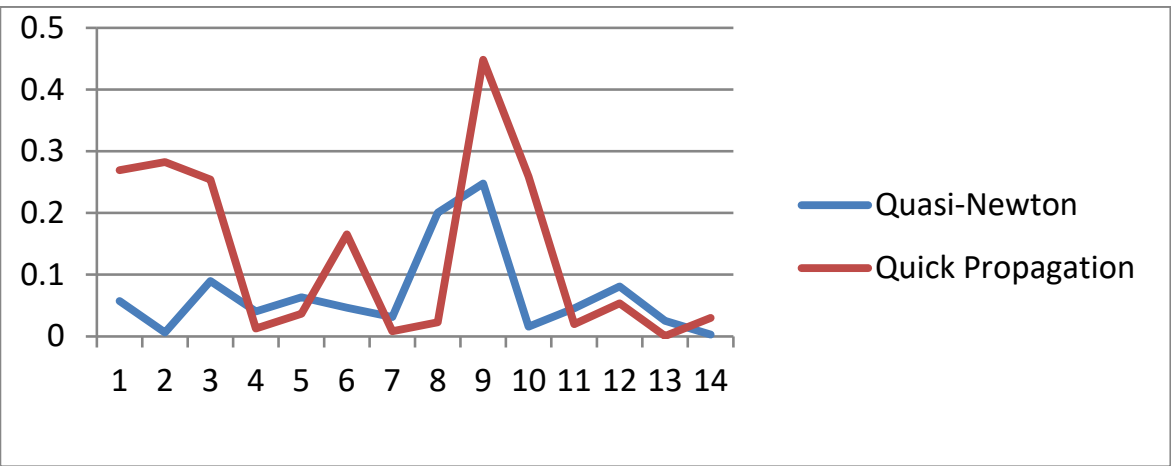

Figure 3: Graph of Quasi-Newton and Quick Propagation algorithms.

(Showing the graph of the two compared ANN training algorithms.)

Quasi-Newton generated a standard deviation of 0.68885 and a $95.46 \%$ correlation while Quick Propagation generated a standard deviation of 0.133047 and a correlation of $90.57 \%$ as shown in Table 2 .

Table 2: Descriptive Statistics

\begin{tabular}{|l|l|l|}
\hline Descriptive Statistics & \multicolumn{1}{|c|}{ Quasi-Newton } & \multicolumn{1}{c|}{ Quick Propagation } \\
\hline Standard Deviation & 0.068885 & 0.13894 \\
Minimum & 0.002927 & 0.000863 \\
Maximum & 0.247961 & 0.448236 \\
Correlation & 0.954575 & 0.905749 \\
\hline
\end{tabular}

Table 3: Fuzzy EOQ and ANN Values Comparison

\begin{tabular}{|l|l|}
\hline FUZZY EOQ & ANN (Quasi-Newton) \\
\hline 31.30 & 31.4 \\
17.30 & 17.31 \\
14.83 & 14.9 \\
3.81 & 3.84 \\
2.90 & 3.0 \\
29.83 & 29.9 \\
4.20 & 4.21 \\
23.70 & 23.90 \\
41.44 & 41.70 \\
15.81 & 15.82 \\
3.63 & 3.65 \\
2.23 & 2.28 \\
4.41 & 4.43 \\
5.50 & 5.51 \\
\hline
\end{tabular}

Results of the experiment show the correlation between the computed outputs (ANN) and the target outputs (Fuzzy logic) is 0.999 when the ANN was trained with the Quasi Newton training algorithms on 100 iterations. This indicates a very strong correlation of the two intelligent tools. The Quasi Newton (QN) method was chosen because when compared with its counterpart the quick back propagation (QBP), it has a correlation value of 0.9546 while QBP has 0.9057 . The mean square error (MSE) for QN is 0.00938 representing $0.94 \%$ while that of QBP is 0.0370 representing $3.7 \%$.

\subsection{Conclusion}

Intelligence is essential in the course of decision making on what quantity is to be ordered and when it is to be ordered. In doing so, an artificial intelligence system is modelled using ANN which is trained on the forecasted data on-demand rate of 15 items collected for ten months in a small and medium scale business retail outlet. With the employment of two independent training algorithms, the Quasi-newton and quick propagation in the training, results show that Quasi-Newton algorithm has a higher converging rate than the quick propagation algorithm with the results found in the literature using fuzzy logic technology. The fuzzy logic results were already compared with the results of the EOQ model and found to covary strongly.

Fuzzy logic is a human-like reasoning technique that is based on approximate rather than the exact reasoning as found in the conventional Boolean logic employed in the EOQ model. ANN is a connectionist system inspired by the biological neurons by mimicking the learning and reasoning capabilities of the human brain. The feedforward architecture using supervised learning paradigm of ANN uses the target output as the teacher through which learning is undertaken. On each iteration, the computed outputs are compared with the target outputs from 
which an error (difference between the target and computed outputs) is computed. In this study, it was observed that there was a convergence of the target outputs with the computed outputs after 100 iterations. The weights that resulted in the convergence represent the knowledge of the system which could be used to compute the optimal quantity to order. With the target outputs computed with a human-like reasoning technique and the training done on ANN and found to correlate strongly, the study justifies the use of an intelligent tool in the prediction of inventory.

Related works found in the literature show that ANN could be used to manage inventory through the training of the networks with average demand datasets. As evident in Paul and Azeem (2011), Farhat and Owayjan (2017), among others. This study enhances the existing work by incorporating the objective and subjective feelings of the inventory manager to complement the results obtained from training. When the filter engine is implemented to supplement the result of the training an optimal results that reflect the economy reality of a particular environment would be obtained. This study also captures, present and future circumstances that were not present in the past and could be used to obtain a realistic and economically feasible quantity to order.

This study is a framework which will could be implemented with the collection of more datasets for training on the networks. Future work will focus on combining the ANN technology with the fuzzy logic forming the kernel.

\section{References}

Agarwal, S. (2014). Economic order quantity model, a review. VSRD Int. J. Mech. Civ. Automob. Prod. Eng, 4(12), 233-236.

Agha, N. C. (2010). Inventory Management and Cost Control in Manufacturing Industries in Nigeria. The Nigeria Journal of management research, 5(2), 173-188.

Agwu, M. O. and Emeti, C. I. (2014). Issues, challenges and prospects of small and medium scale enterprises (SMEs) in Port-Harcourt city. European Journal of Sustainable Development, 3(1), 101-114.

Akay M. F., Zayid E. M., Akturk, E and George J. D. (2011). Artificial Neural Network-Based Model for Predicting $\mathrm{VO}_{2}$ ax rom a submaximal Exercise Test. Expert System with Applications 38(2011), 2007-2010

Anwana, E. and Nyah, D. (2017). Quantitative Techniques for Business and Management. Ritman University Press, Ikot Ekpene, Nigeria.

Basil, A. N. O. (2005). Small and Medium Enterprises (SMES) in Nigeria: Problems and Prospects, A Ph.D. Thesis. St. Clements University, UK.

Bansal K. and Vadhavkar S. and Gupta, A. (1997). Brief Application Description of Nueral Networks Based Forecasting Techniques for Inventory Control Application. Data Mining and Knowledge Discovery, 2(1998), 97-102.

Boru, A., Dosdogru, A. T., Goçken, M. and Erol, R. (2019). A Novel Hybrid Artificial Intelligence Based Methodology for the Inventory Routing Problem, Symmetry 2019, 11, 717; doi:10.3390/sym11050717.

Damirchi, Q.V. and Rahimi, G. (2011). Design a conceptual ERP model for small and medium enterprises of Iran. Interdisciplinary Journal of Contemporary Research in Business, 3(5), 850-861.

Dong X. and Wen G. (2006). An Improved Neural Networks Prediction Model and its Application in Supply Chain. Nature and Science, 4(3), 23-27.

Eze, T.C. and Okpala, C.S. (2015). Quantitative analysis of the impact of small and medium scale enterprises on the growth of Nigerian economy:(1993-2011). International Journal of development and emerging economics, 3(1), 26-38.

Farhat, J. and Owayjan, M. (2017). ERP Neural Network Inventory Control. Procedia computer science, 114, pp.288-295.

Gonzalez, J. L., \& Gonzalez, D. (2010). Analysis of an Economic Order and Reorder Point Inventory Control Model. A B.Sc Project of the Department of Industrial Engineering. California Polytechnic State University, San Lius Obispo, USA.

Ihua, U.B. (2009). SMEs key failure-factors: a comparison between the United Kingdom and Nigeria. Journal of Social Sciences, 18(3), 199-207. Iss 1, 155 - 174.

Jabłonska, M. R., Pólkowski, Z. (2017). Artificial Intelligence-Based Processes in SMEs, Studies \& Proceedings of Polish Association for Knowledge Management, Nr 86, 2017.

Kehinde, O.J. (2016). Small and medium scale enterprises: Pivotal to sustainable economic development: The Nigerian experience. International Journal of Current Research, 8(1), xxx-xxxx.

Kosiński, W., Muniak, R. and Kosiński, W.K. (2013). A model for optimizing enterprise's inventory costs: a fuzzy approach. Operations Research and Decisions, 4, 39-54.

Kotler, P. (2000). Marketing management: The millennium edition. Marketing management, 23(6), 188-193.

Kumar, N. and Kumar, S. (2017). An inventory model for deteriorating items with partial backlogging using linear demand in fuzzy environment. Cogent Business \& Management, 4(1), 1307687.

Llave, M. R. (2017). Business Intelligence and Analytics in Small and Medium-sized Enterprises: A Systematic 
Literature Review, Procedia Computer Science 121 (2017), 194-205.

Motilewa, D.B. (2015). A review of the impacts SMEs as social agents of economic liberations in developing economies. International Review of Management and Business Research, 4(3), 903-914.

Muller, M. (2019). Essentials of inventory management. HarperCollins Leadership.

Obot, O.U., George, U.D. and Umana, V.S. (2019). A Decision Support Tool (DST) for Inventory Management. International Journal of Decision Support System Technology (IJDSST), 11(2), 27-47.

Ogundele, O.J.K. et al. (2013). Marketing practice of small and medium enterprises (SMEs): Perspective from a developing country. Mediterranean Journal of Social Sciences, 4(3), 243.

Oluboba, O. (2011). "Career Crises and Financial Distress: The Way Out", in Joseph Ajayi, J. and Adesina, S. "Experts Highlight Problems Militating against SMEs in Nigeria". Tribune Business. Wednesday, 16 February 2011 .

Pan F. (2016). Inventory Prediction Research Based on the Improved BP Neural Network Algorithm. International Journal of Grid and Distributed Computing (9), 307-316.

Paul, S.K. and Azeem, A. (2011). An artificial neural network model for optimization of finished goods inventory. International Journal of Industrial Engineering Computations. 2(2), 431-438.

Ramli, A., Jusoh, I. and Ali, M.R.M. (2017). A combination of Broyden-Fletcher-Goldfarb-Shanno (BFGS) and $\mathrm{n}$-th section method for solving small-scale unconstrained optimization. Malaysian Journal of Fundamental and Applied Sciences, 13(4), 717-720.

Taiwo, M.A., Ayodeji, A.M. and Yusuf, B.A. (2012). Impact of small and medium enterprises on economic growth and development. American journal of business and management, 1(1), 18-22.

Sekar T. and Uthayakumar, R. (2018). A Production Inventory Model for a Single Vendor and Single Buyer Integrated Demand with Multiple Production Setups and Rework. Uncertain Supply Chain Management, 6(2018), 75-90.

Schuller, D. and Doubravsky, K. (2019). Fuzzy Similarity Used by Micro-Enterprises in Marketing Communication for Sustainable Development, Sustainability 2019, 11, 5422; doi:10.3390/su11195422.

Zigiene, G., Rybakovas, E. and Alzbutas, R. (2019). Artificial Intelligence Based Commercial Risk Management Framework for SMEs, Sustainability 2019, 11, 4501; doi:10.3390/su11164501 